\title{
Spatial-temporal Pattern of Regional Economic Disparities in GuiYang
}

\author{
$\mathrm{YinFu}^{1}$, WeiShui ${ }^{1{ }^{* *} \text {, }}$ \\ ${ }^{1}$ College of Environment and Resources, Fuzhou Unviersity, FuZhou, P.R.China;
}

\begin{abstract}
Based on the construction of indicator system for evaluating Comprehensive index of economic development, the synthetic economic development levels are evaluated from 2000 to 2015 in GuiYang. GIS Spatial analysis has studied the disparities in economic development in GuiYang. By calculating the spatial autocorrelation Moran's I index of GuiYang Comprehensive Development Index, it reveals that it's economic development has significant spatial autocorrelation, and the regional economic development has obvious dual structure; finally, through analyzing the local indicator of spatial association (LASA) each year. this paper reveals the temporal and spatial variation characteristics of economic development in GuiYang.
\end{abstract}

\section{Introduction}

Since the implementation of the great development of western china in 2000, GuiYang has become an important central city and transportation hub in the southwest region, and it's economy has developed rapidly. In 2017, the regional GDP reached 353.796 billion yuan, an increase of 11.68 times over 2000. However, the economic development of GuiYang City also has a growing regional difference. According to the theory of regional economic development, appropriate economic development disparities can increase regional competition and promote regional development. However, when economic disparities between regions are too bigger, it will affect regional stability and solidarity, even our Nation's Prosperity ${ }^{[1]}$. Therefore, timely and scientifically evaluating regional economic development and narrowing economic development disparities are of great significance for realizing regional economic development and even national prosperity. At present, scholars have done a lot of research on the economic development status, spatial distribution and it's influencing factors in GuiYang City, but most of them are based on single indicator such as per capita income gap of urban residents ${ }^{[2]}$ and county per capita GDP ${ }^{[3]}$, there are few studies based on multi-indicator comprehensive development index. Therefore, this paper constructs a multi-index system and compute economic development comprehensive Index and uses GIS spatial analysis method to study it's humidity and wind on heat exposure. Therefore, our research explored the spatial characteristics of heat exposure in bay cities by integrating multi-climate and population factors, in order to provide targeted decision support for humans to cope with future high temperature risks.economic development difference.

\section{Research methods}

(1) Principal component analysis

This paper constructs an evaluation index system from four aspects: economic scale, economic benefit, economic structure and people's life. Principal component analysis method is used to determine the weight of each index, and further calculate the economic development comprehensive index, in order to evaluate the economic development disparities of every area in GuiYang. The formula as follow:

$$
Z=\sum X_{I} * W_{J}
$$

$X_{I}$ as the data of various economic indicators that are standardized, and $\mathrm{W}_{\mathrm{J}}$ as the weight of each indicator.the weight of each indicator.

(2) global spatial autocorrelation analysis

Global spatial autocorrelation is to analyze the spatial distribution of a given object in the entire study area to determine whether it has spatial autocorrelation. The indices describing the global spatial autocorrelation are Moran's I, Geary's C, etc. We mainly use the Moran's I index, which reflects the similarity of the values of the spatial neighbors or spatial neighbors between the research units.The Moran index ranges from $[-1,1]$, and Moran's I $>0$ represents spatial positive correlation. The higher the value the stronger spatial correlation is Moran's $\mathrm{I}<0$.

\footnotetext{
*Corresponding author:2909296476@qq.com
} 
Tab.1 The indicator system of spatial economic development level

\begin{tabular}{|c|c|}
\hline & Indicator Describe \\
\hline $\begin{array}{l}\text { economic } \\
\text { structure }\end{array}$ & $\begin{array}{l}\text { The proportion of secondary industry } \\
\text { The proportion of first industry }\end{array}$ \\
\hline Economic Benefit & $\begin{array}{l}\text { Per Capital GDP } \\
\text { Per capital social fixed assets } \\
\text { Per capital social consumption goods } \\
\text { Per capital social investment in fixed assets }\end{array}$ \\
\hline economic scale & $\begin{array}{l}\text { Gross regional product GDP } \\
\text { urban and rural residents savings } \\
\text { Import and Export Total }\end{array}$ \\
\hline people's lives & $\begin{array}{l}\text { per capital disposable income of urban residents } \\
\text { Per capital urban and rural resident deposits } \\
\text { Net Income of Rural Households }\end{array}$ \\
\hline
\end{tabular}

represents the spatial negative correlation, and the smaller the value the spatial difference is also stronger, and when Moran's $\mathrm{I}=0$, the spatial distribution is random and has no correlation. The expression formula of the global Moran's I is as follows:

$$
I=\frac{n}{\sum(x+-x)} \times \frac{\sum_{i=1}^{n} \sum_{j=i}^{n} W_{i j}(x,-X)(X,-X)}{\sum_{i=1}^{n} \sum_{j \neq i}^{n} W \|} \text { (2) }
$$

In the above formula, $\mathrm{n}$ represents the number of cells of the study area, $\mathrm{W}_{\mathrm{ij}}$ represents the spatial matrix of the region $i$ and the region $j$, and $X_{i}$ and $X_{j}$ represent the observed value of the region $i$ and the region $j$. According to the Moran index calculated from the data, a Z-value test is also performed to Verify whether there is spatial autocorrelation between the $\mathrm{N}$ regional units studied.The expression formula for $\mathrm{Z}$ is as follows:

$$
Z=\frac{I-E(I)}{\sqrt{\operatorname{var}(I)}}
$$

In the above formula, the expected value of the $E(I)$ bit, $\operatorname{var}(\mathrm{I})$ is the variance, and in addition to the $\mathrm{Z}$-value test, a P-value test is also performed.

(3) Local spatial autocorrelation analysis

The global spatial autocorrelation can reflect the high and low distribution correlation of regional economic development disparities globally, but it does not reflect the specific location of the economic development between local units under the overall unit and the degree of correlation between the regions. Local spatial autocorrelation is to analyze whether the local area specified attribute has spatial autocorrelation. The methods for describing local spatial autocorrelation are Local Moran's I (LISA) and Moran scatter plots ${ }^{[4]}$.

\section{3 results and analysis}

\subsection{Analysis of the time characteristics of economic development in GuiYang City}

The statistical data of various economic indicators in 2000, 2005, 2010 and 2015 were brought into the formula to calculate comprehensive economic development index of respective period. The maximum and minimum value and standard deviation of comprehensive economic development index was calculate by SPSS across years. The results are as follows: Ranking by the index, YunYan District ranked first in economic development among GuiYang cities in 2000, 2005 and 2010 because it is the center of GuiYang, with population and industry clusters and convenient transportation. With the development of NanMing District, NanMing has become another center of GuiYang By 2015, the economic development of NanMing District ranked first. And KaiYang County, XiFeng County, XiuWen County and QingZhen City are always ranking last four place, which are located in the edge of GuiYang with insufficient resources and manpower and the inconvenient traffic. In 2000, From the time variation of the index (Fig.2 and Table 2), the maximum value is 4.53 in YunYan area, and the minimum value is 2.23 in XiuWen County; the range is 6.76, and the standard deviation is 2.09. In 2005, the maximum value is 3.99 in YunYan area, the minimum value is -2.30 in XiuWen ; the range is 6.29 , and the standard deviation is 2.08. In 2010, the maximum value is 4.20 in YunYan, the minimum value is -2.21 in XiuWen; the range is 6.49 , and the standard deviation is 1.97. In 2015, the maximum value is 2.89 in NanMing, the minimum value is -2.16 in XiuWen; the range is 5.05, and the standard deviation is 1.91 .

The range was greatly reduced by 0.47 from 2000 to 2005 , indicating a period that the economic development gap across provinces are gradually shrinking. From 2005 to 2010 , the standard deviation slightly increased by 0.2 , indicating a stage of enlarging economic development gap. From 2010 to 2015 has decreased by 0.44 , indicating a period that economic development gap was narrowing. In general, the economic development of GuiYang City has experienced a process of shrinking, expanding and then shrinking again, showing a typical "U"-shaped change trend.

\subsection{Global Spatial Autocorrelation of Comprehensive Indexes of Economic Development}

The spatial autocorrelation coefficient of the economic 
development comprehensive index of GuiYang City in 2000, 2005, 2010 and 2015 was calculated by GeoDA software, indicating that the positive economic autocorrelation of GuiYang City's economic development from 2000 to 2015, It shows that the level of economic development around the regions with high economic development index is also high, that is, they are clustered together, and vice versa. For example, the Moran's I index of 2015 is 0.516 , which reveals that the economic development of GuiYang City has significant spatial agglomeration in this year. This phenomenon is consistent with the

Table 2 .The evaluation and analysis of economic development level in GuiYang

\begin{tabular}{ccccccc}
\hline year & $\mathrm{N}$ & minimum value & maximum value & Mean Value & Standard Deviation & range \\
\hline 2000 & 12 & -2.23 & 4.53 & 0.084 & 2.09 & 6.76 \\
2005 & 12 & -2.3 & 3.99 & 0.002 & 2.08 & 6.29 \\
2010 & 12 & -2.29 & 4.2 & 0.001 & 1.97 & 6.49 \\
2015 & 12 & -2.16 & 2.89 & 0.003 & 1.91 & 5.05 \\
\hline
\end{tabular}
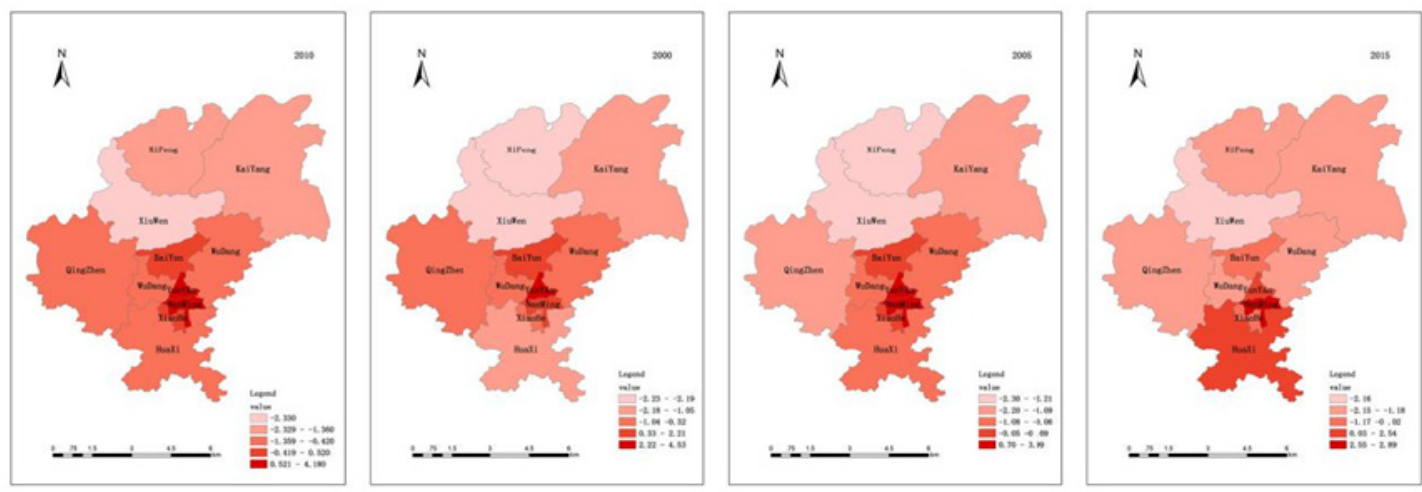

Fig. 1. Distribution of comprehensive economic development indices of various regions in GuiYang

hierarchical map of economic development comprehensive index shown in Figure 2. In 2010, the value of the Moran's I index in GuiYang City fluctuated greatly, from 0.4268 in 2005 to 0.3594 in 2010 . Since then, it has resumed it's growth trend and increased to 0.516 in 2015 , but the spatial autocorrelation in this period has always been maintained. At a higher level, it indicates that the regional economic development of GuiYang City has slowed down during this period. To a certain extent, it shows the rapid development of the suburb of GuiYang, such as HuaXi. It shows that the rapid development of urbanization in GuiYang has gradually transitioned to suburbanization. Stage. In general, the Moran's I index of GuiYang City Mainly located in "high-high", "low-low" and two quadrants, and the annual fluctuations are not large. It shows that since 2000, the regional disparities in economic development of GuiYang City have shown a clear spatial dual structure. The hot spots of economic development are mainly in YunYan District and NanMing District of GuiYang City. and the marginal areas of XiFeng County, XiuWen County and Kai Yang is in a blind spot of economic development, which is consistent with the reality.

\subsection{Partial autocorrelation of GuiYang Economic Development Comprehensive Index}

From the Moran's I index scatter plot, it can be clearly seen that the spatial characteristics of economic development in GuiYang show a distinct spatial dual structure, The prefecture-level cities such as YunYan District, NanMing District, KaiYang County and
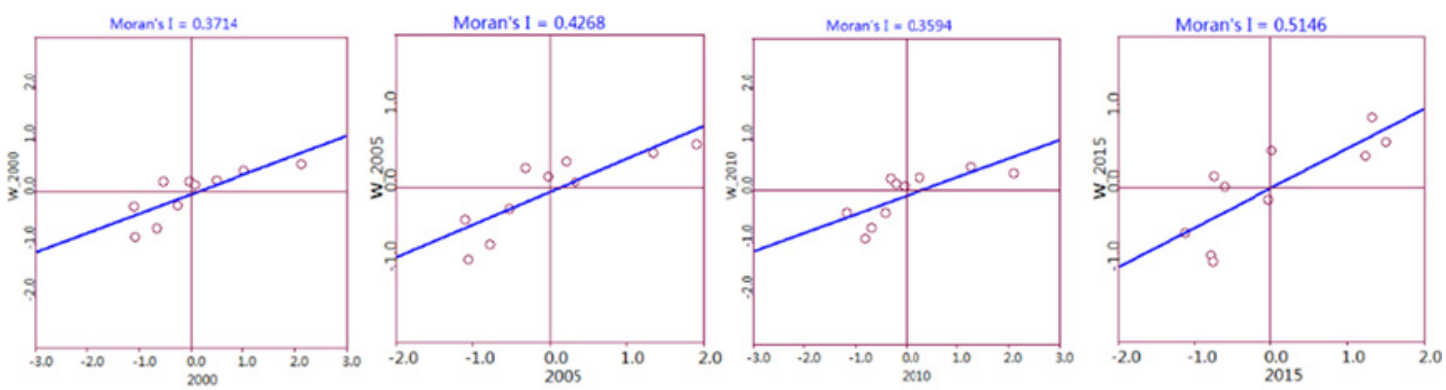

Fig. 3. Spatial autocorrelationMoran's I index of economic development in various regions of GuiYang City

XiuWen County, which belongs to the "High to High"and "Low to Low" quadrants, has strong spatial positive correlation. But did not indicate the degree of spatial autocorrelation in each region. The LISA value compensates for this deficiency, which 
is a unit property that measures the similarity and dissimilarity of spatial unit properties to surrounding elements. Use the GeoDA software to calculate the LISA value of the 2000-2015 economic development comprehensive Index of every area in GuiYang, and plot the LISA values for each year, as shown in Figure 3. With the acceleration of the urbanization process in GuiYang, the radiation range of the city center has gradually expanded, from the original downtown Yunyan District and NanMing District to the surrounding areas of HuaXi District, XiaoHe District, WuDang District and Baiyun District, revealing this During the period, the hot spots of economic development were mainly in the YunYan and NanMing districts and adjacent neighboring cities, and their ability to radiate outward was slightly insufficient. The four prefecture-level cities of KaiYang County, XiFeng County, XiuWen County and QingZhen City have always been "low to low" areas, and have not yet entered the "High to High" area, indicating that the economic development of the downtown YunYan District and NanMing District has not yet Can be extended to the region, the
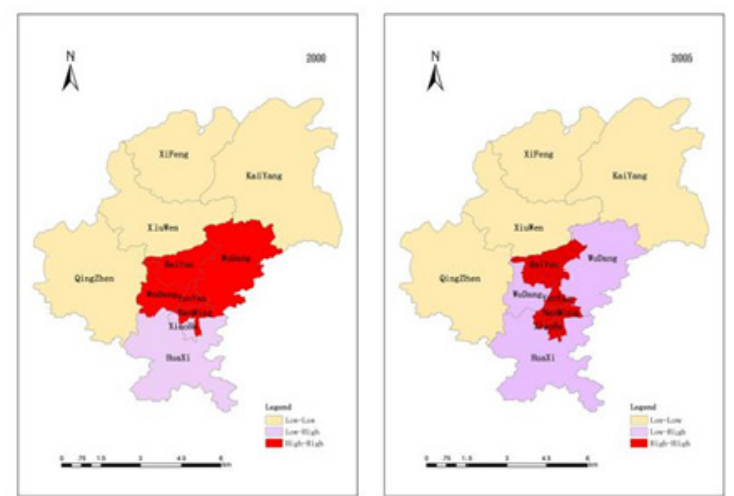

Fig. 4. GuiYang Economic Development Comprehensive Index LISA Aggregation Chart

autocorrelation. mong them, YunYan District and NanMing District are the core areas of economic development in GuiYang. The economic development level of XiFeng County, KaiYang County, XiuWen County and QingZhen City in the marginal areas is low, indicating that GuiYang City is still in a period of rapid expansion., no suburbanization

\section{References}

1. Zhou Y C, Qing-Wen Q I, Feng C F. (2002), Characteristics of dynamic variation of the inter-provincial economic difference in China in recent ten years[J]. Geogr Res 21(6):781-790.

2. Yi-Guang Y E, Wang Q F(2003). Analysis on the change of regional economic difference in Jiangsu Province since economic reform[J]. J Nanjing Agric Univ.

3. Liu Q C, Wang Z.(2009), Research on geographical elements of economic difference in China[J].Geogr Res, 28(2):430-440.

4. Niu L, Luo W, Jiang M, et al.(2018) Land-Use Degree and Spatial Autocorrelation Analysis in Kunming City Based on Big Data[C]// International Conference on Intelligent Transportation, Big Data \& Smart City. IEEE Computer Society:97-100.
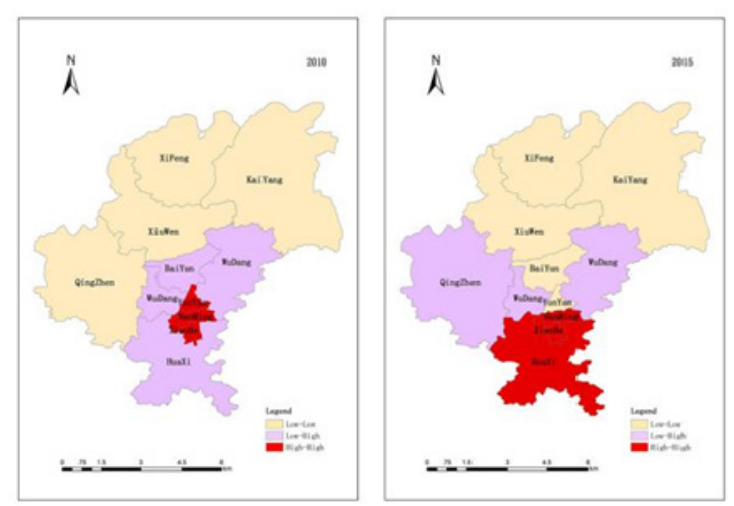

urbanization of GuiYang is still in an accelerated development period, and there is still a suburbanization.

\section{4 conclusion}

This paper briefly introduces the technical characteristics of GIS based spatial analysis method, and empirically studies the regional disparities of economic development in GuiYang based on the spatial autocorrelation method. revealing the spatial and temporal changes in regional development differences in Guiyang City. The results show that the economic development of GuiYang City from 2000 to 2015 experienced a "U"-type change trend of first shrinking, then expanding and then shrinking, and it's economic development showed significant spatial . 